\title{
Molecular phylogeny, biogeographic history, and evolution of cave-dwelling taxa in the European harvestman genus Ischyropsalis (Opiliones: Dyspnoi)
}

Axel L. Schönhofer ${ }^{1,2}$, Cristiano Vernesi ${ }^{3}$, Jochen Martens ${ }^{2}$ and Marshal Hedinn ${ }^{1}$. ${ }^{1}$ Department of Biology, San Diego State University, San Diego, CA 92182-4614, USA. E-mail: mhedin@mail.sdsu.edu; ${ }^{2}$ Institute of Zoology, Johannes Gutenberg University Mainz, D-55099 Mainz, Germany; ${ }^{3}$ Department of Biodiversity and Molecular Ecology, Centre for Research and Innovation-Fondazione Edmund Mach, San Michele all'Adige 38010 (TN), Italy

\begin{abstract}
We estimated a multigenic molecular phylogeny and reconstructed biogeographic history for the European harvestman genus Ischyropsalis C.L. Koch 1839 (Dyspnoi). To reconstruct historical biogeographic patterns we conducted an algorithmic VIP analysis which revealed patterns consistent with a vicariance-dominated history. The existing morphology-based systematic framework for Ischyropsalis is mostly inconsistent with molecular phylogenetic results, and a new informal system is established that recognizes three main clades and several sub-clades. Species-level analyses revealed two non-monophyletic species (I. pyrenaea Simon 1872 and I. luteipes Simon 1872); subspecies of I. pyrenaea are distant relatives, and are formally elevated to species (I. pyrenaea pyrenaea to I. pyrenaea and I. pyrenaea alpinula to I. alpinula). A preference for cryophilic microhabitats has favored the diversification of high-altitude and cavedwelling Ischyropsalis species; molecular phylogenetic data suggest that cave-dwelling species have evolved multiple times independently.
\end{abstract}

Keywords: Species tree, multilocus phylogeny, biogeographic modelling, convergence, ecological specialization, cave evolution

Modern biogeographic studies of harvestmen have revealed an interesting mixture of evidence for extremely limited dispersal (e.g., Thomas \& Hedin 2008; Derkarabetian et al. 2011; Boyer \& Reuter 2012), combined with evidence for occasional long-distance dispersal (e.g., Shultz \& Regier 2009; Sharma \& Giribet 2012; Schönhofer et al. 2013). As such, harvestmen are compelling systems for biogeographic research, but as expected many harvestmen taxa remain to be investigated using modern phylogenetic and biogeographic methods. Within the European fauna, members of the genus Ischyropsalis C.L. Koch 1839 are among the most charismatic harvestmen, with relatively large body sizes and massively enlarged chelicerae used to catch prey (Fig. 1). Ecologically, members of this genus are often rare and found in difficultto-access habitats, including several obligate cave-dwelling species.

Ischyropsalis species limits have been investigated in the context of the biological species concept (Mayr 1942), rarely explicitly applied within arachnids. Martens (1969a) observed Ischyropsalis males offering females a secretion from the basal cheliceral segment during courtship and copulation, and demonstrated an association between the secretion offering and subsequent contact between this secretion area and the female mouthparts (Martens 1969b). The male cheliceral secretion area includes bristle fields (Fig. 1) that are connected by minute pores to massive glands (Martens \& Schawaller 1977). Martens (1969b) made this cheliceral secretion area and the resulting associated male/female interaction a fundamental character to delineate Ischyropsalis species, and also found corresponding male genital characteristics. The presence of such character combinations has also been adopted for species delimitation and higher-level classification in other Opiliones, for example in the Nemastomatidae (Schönhofer \& Martens 2012), Dicranolasmatidae (Gruber 1998) and Sabaconidae (Martens 1972; Suzuki 1974).
Studies of Ischryopsalis classification began with Hadži (1931), who delineated a single Ischyropsalis subgenus, Odontopalpa, based on apophysis characters on the male palpal patella (Fig. 1). Originally containing three species, two species were synonymised (Martens 1969b) with I. kollari C.L. Koch 1839 (I. bosnica Roewer 1914, I. triglavenis Hadži 1931), the type species of Ischyropsalis. As it was not considered in Odontopalpa, the subgenus then became invalid. Hadži originally also placed I. dentipalpis Canestrini 1872 and later I. ravasinii Hadži 1942 within Odontopalpa but had no males to confirm the male-specific characters of this subgenus for the latter. Roewer (1950) described numerous new Ischyropsalis species, but was suspected to have separated specimens of common species to describe new species. Martens (1969b) detected these manipulations, later substantiated by $\mathrm{v}$. Helversen and Martens (1972), and synonymized 33 of 34 of Roewer's species.

Morphology-based phylogenetic hypotheses for Ischyropsalis were published nearly simultaneously by Martens (1969b) and Dresco (1970). Dresco drew his conclusions from the Paris Museum collection, containing mostly Iberian Ischyropsalis, while he had limited access to and knowledge of the remaining European fauna. Martens (1969b) considered most currentlyaccepted species, and provided an extensive revision based on characters newly developed for Ischyropsalis systematics. The systems of Martens and Dresco only agree in the isolated position of I. hellwigii (Panzer 1794) while other hypothesised groupings show little congruence (Fig. 2). Authors after 1970 did not comment on the phylogenetic structure of the genus, but added new species or further clarified species synonymies. Martens (1978) refined his system for some Alpine species. Luque (1991, 1992) and Prieto (1990a, b) described the Spanish fauna in more detail. Most recently, Schönhofer and Martens (2010a) and Luque and Labrada (2012) added two new species and discussed regional species diversity in the 

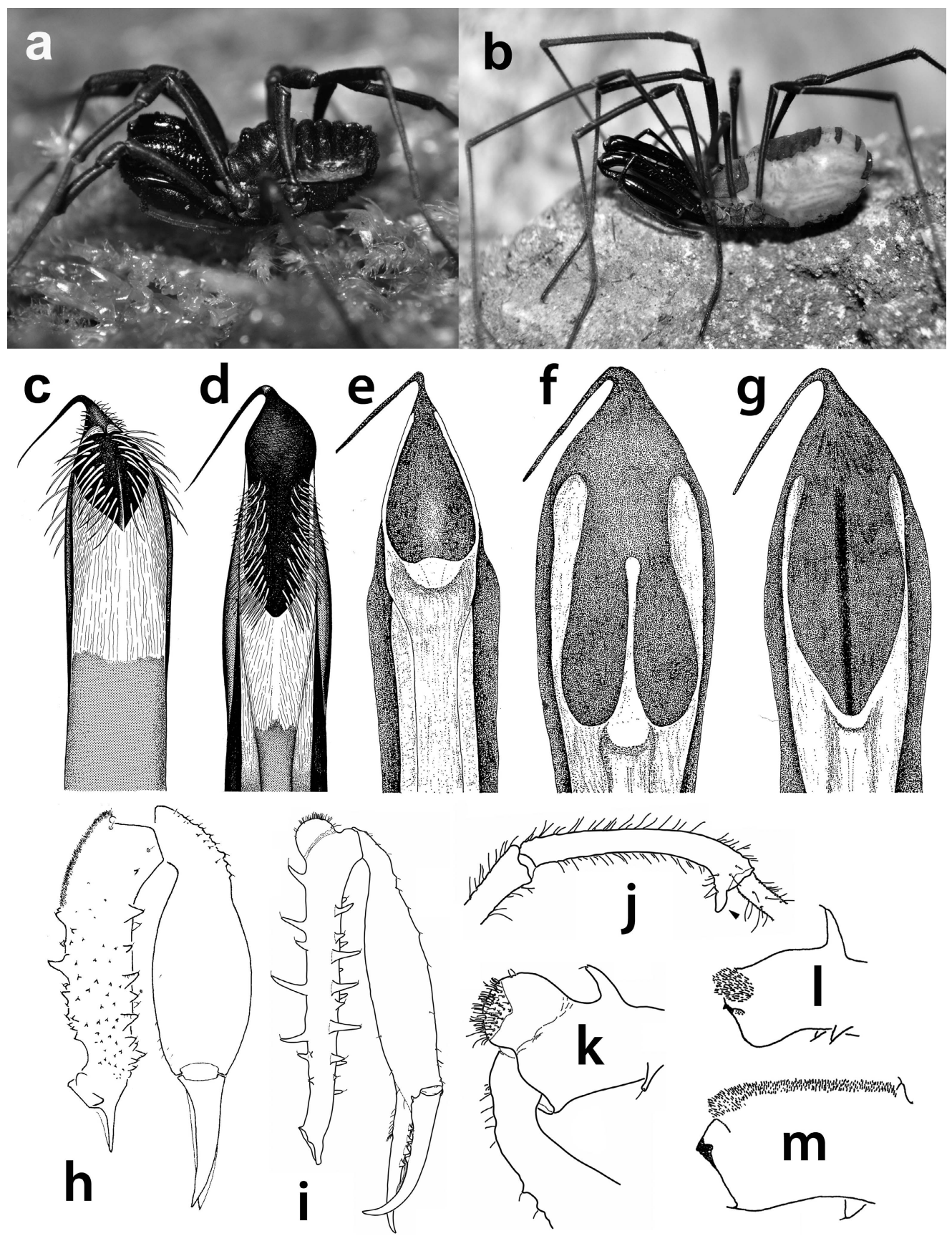

Figure 1.-Ischyropsalis habitus and morphological features. a) I. hellwigii hellwigii male habitus, Austria, Soboth; b) I. pyrenaea pyrenaea female habitus, France, Grotte de l'Estelas; c) distal part of penis, I. pyrenaea alpinula (Martens 1978; Fig. 381); d) distal part of penis, I. dentipalpis (as I. helvetica; from Martens 1978: Fig. 382); e-g) different types of glans sclerites (groups sensu Martens, 1969b; Fig. 32): e) I. hellwigii group, f) I. kollari group, g) I. dentipalpis group; h, i) male cheliceral armament, h) I. hellwigii hellwigii (Martens 1978: Fig. 289), i) I. dentipalpis (Schönhofer \& Martens 2010a); j) apophysis on palpal patella of male I. dentipalpis (Schönhofer \& Martens 2010a; Fig. 6); k-m) distal part of male chelicerae (medial view), apophyses and cheliceral secretion-extruding-field indicated by bristlefield: k) I. lithoclasica (Schönhofer \& Martens 2010a; Fig. 13), 1) I. adamii, m) I. hellwigii (both Martens 1969b; Fig. 22).

Western Alps and the Cantabrian Mountains, respectively. Twenty-two species-level taxa are currently recognized in the genus (Schönhofer 2013a).

Based on the revision of Martens (1969b), distributional patterns in Ischyropsalis suggest a complex biogeographic history. The three species groups of Martens (1969b) are widespread and overlap in distribution, while species within groups are strictly allopatric. This broad-scale phylogenetic structure and the proposed species inter-relationships imply repeated dispersal and isolation between the major mountain systems in Western and Central Europe. On the other hand, individual Ischyropsalis species have generally narrow distributions, and at least nine species are obligate cave endemics. Cave-dwelling species are unlikely to disperse over large 


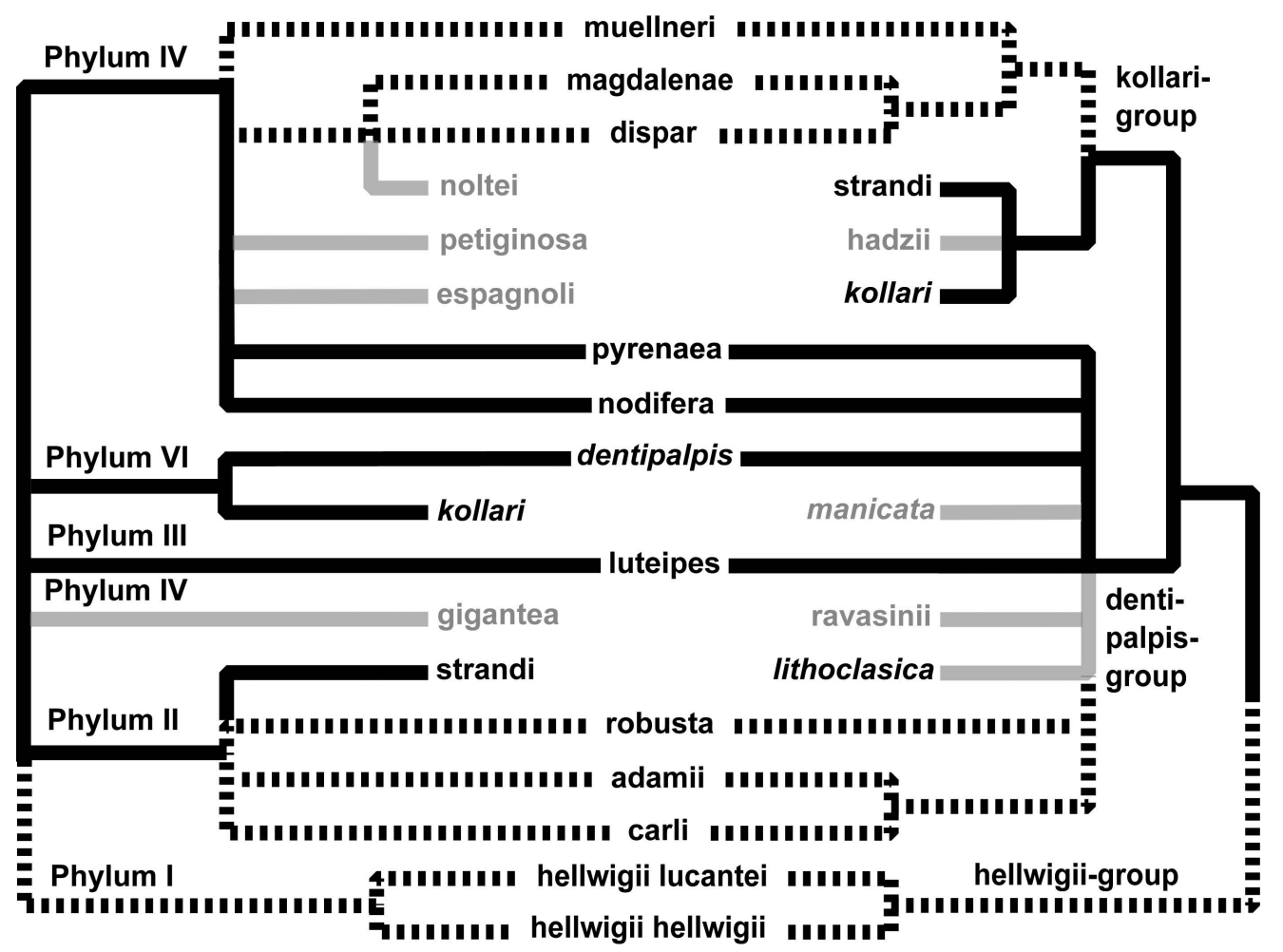

Figure 2.-Morphology-based phylogenetic hypotheses for Ischyropsalis according to Dresco (1970, left) and Martens (1969b, right). Some synonymies / misinterpretations used by Dresco were replaced for ease of comparison (we use I. muellneri for I. hellwigii, I. hellwigii hellwigii for I. taunica, I. adamii for I. apuanus, I. kollari for I. trigalvensis and I. hellwigii lucantei for I. superba). Phylogenetic lineages are compared and matched if their taxa composition shows at least two matches agreed upon by both authors; matching taxa are indicated on dashed branches; taxa corresponding to alternative lineages are indicated on black branches; taxa considered by only one author are designated in grey. Nonmatching species are separated by space between the species names. Species of subgenus Odontopalpa sensu Hadži (1931) shown in italics.

distances, and may have originated from widespread epigean species (Porter 2007).

We present here the first molecular phylogenetic analysis of Ischyropsalis to investigate the validity of alternative higherlevel classification schemes as well as multiple species and subspecies hypotheses (Martens 1969b; Dresco 1970). This molecular phylogenetic framework also allows us to address the phylogenetic value of currently used morphological characters-because of their relatively simple organization and structure, Martens (1969b) suggested the possibility of morphological convergence in male genitalia of Ischyropsalis. The distribution of all Ischyropsalis taxa is reconstructed based upon literature, museum collections and personal collection data, and these data are used in algorithmic analyses to reconstruct Ischyropsalis biogeographic history. Finally, molecular phylogenetic results provide insight into whether cave-dwelling taxa originate from a single ancestor, or have evolved independently multiple times.

\section{METHODS}

Sampling.-Ischyropsalis specimens were collected by hand, mainly during our own field excursions. Some specimens were also generously donated by colleagues (see Acknowledgments). Specimens were preserved in $100 \% \mathrm{EtOH}$, with the majority being collected in $80 \%$ and transferred soon thereafter. Morphological identifications were based on
Martens (1969b, 1978), Luque (1991, 1992) and Schönhofer and Martens (2010a), or provided by C. Prieto for Spanish samples. A few specimens were raised to adulthood in captivity to allow morphological species identification. We included as many described species as possible, and conducted intraspecific sampling to include geographically and morphologically distinct lineages. Voucher specimen details and repository information are provided in the Appendix. Voucher specimens (with associated collection numbers) are deposited in the collections of J. Martens (CJM, Mainz, Germany), C. Prieto (CCP, Bilbao, Spain), A. Schönhofer (AXLS, Mainz, Germany), C. Vernesi (FEM: Centre for Research and Innovation-Fondazione Edmund Mach, San Michele all'Adige, Italy) and the San Diego State University Terrestrial Arthropod collection (OP).

Molecular methods and analyses.-Genomic DNA was extracted from tissue using the Qiagen DNeasy kit (Valencia, CA, USA). The polymerase chain reaction (PCR) was used to amplify the following gene fragments: 28S rRNA (28S), using the primers ZX1elong (Schönhofer et al. 2013), ZX1, and ZR2 (Mallatt \& Sullivan 1998); mitochondrial Cytochrome Oxidase 1 (CO1), using C1-J-1718SPIDERA and C1-N-2776SPIDER (Vink et al. 2005); and nuclear Elongation Factor 1-alpha $($ EF1 $\alpha$ ), using OP2BSAB and OPRC4 (Hedin et al. 2010). PCR protocols followed Hedin et al. (2010) for EF1 $\alpha$, Thomas and Hedin (2008) for CO1, and Hedin and Thomas (2010) for $28 \mathrm{~S}$, the latter using an annealing temperature of $56^{\circ} \mathrm{C}$. 
Amplicons were purified on Millipore plates and directly Sanger sequenced at Macrogen USA in both directions. SEQUENCHER v4.5 was used to assemble and edit sequence contigs, with ambiguous sites scored using standard ambiguity codes.

CO1 and EF1 $\alpha$ exon sequences were aligned manually in Mega 4.0 (Tamura et al. 2007) using amino acid translation, while $28 \mathrm{~S}$ and EF1 $\alpha$ intron sequences were aligned with MAFFT vers. 6 (http://mafft.cbrc.jp/alignment/software/), using the Q-INS-i strategy as recommended by Katoh and Toh (2008). To account for alignment uncertainty in the $28 \mathrm{~S}$ and the EF $1 \alpha$ intron data, outgroup genera were removed and the data were realigned using the same strategies as above. Models of DNA sequence evolution were evaluated using jModelTest 0.1.1 (Posada 2008) under three substitution schemes (JC, HKY, GTR) on a fixed BIONJ tree, allowing for unequal base frequencies and among-site rate variation. Final model selection was based on the Akaike Information Criterion (AIC) and individual models were applied to respective partitions in all downstream phylogenetic analyses.

Bayesian inference using MrBayes v3.2.0 (Huelsenbeck \& Ronquist 2001; Ronquist et al. 2011) was applied to single genes as well as concatenated datasets. DNA sequences were partitioned by gene, EF1 $\alpha$ by exon and intron, and CO1 and EF1 $\alpha$ exons by codon position. Bayesian analyses were run for 5 million generations, where in all cases the standard deviation of split frequencies had dropped below 0.01 and standard convergence diagnostics were satisfied for all sampled parameters (ESS $>200$, PSRF $=1.00$; Ronquist et al. 2011). Analyses were repeated to further check for convergence. The first $40 \%$ of trees were discarded as burn-in, with remaining trees used to reconstruct a maximum clade credibility tree. Split frequencies were interpreted as posterior probabilities (pp) of clades. A maximum likelihood (ML) concatenated tree using the partitioning scheme outlined above was reconstructed with the raxmlGUI (Stamatakis 2006; Silvestro \& Michalak 2012), with support assessed using 1000 rapid bootstrap replications.

As an alternative to concatenation, a species tree was reconstructed using the multispecies coalescent model implemented in *BEAST (Heled \& Drummond 2010; Drummond et al. 2012). Models of molecular evolution suggested by jModelTest were implemented, with codon partitioning applied to $\mathrm{CO} 1$ and $\mathrm{EF} 1 \alpha$ exons $((1+2), 3)$. Priors were set to gamma if modified, otherwise default priors were used. Analyses were run until ESS values exceeded 200 for most priors, which was after 200,000,000 generations. Analyses were replicated twice and checked for convergence using Tracer v1.5 (Rambaut \& Drummond 2007).

Species trees were estimated using two different sets of operational taxonomic units (OTUs). The first set included only described and accepted species and subspecies (= conservative species set), while the second set was extended to include additional genetic lineages within the species $I$. adamii Canestrini 1873, I. dentipalpis and I. luteipes Simon 1872 recovered in concatenated analyses $(=$ diversified species set). Preference for one of these OTU settings was evaluated by comparing marginal likelihoods using Bayes Factor analysis (Grummer et al. 2014), as implemented in Tracer v1.5 (Rambaut \& Drummond 2007).
Biogeographic analysis.-We compiled geographic distribution data for all known Ischyropsalis taxa. A total of 1,360 distribution records were extracted from literature, museum and personal collections, of which 1,220 could be georeferenced at a minimum accuracy of two digital degrees. Over 1,100 coordinates matched species and geographic lineages in our dataset and were used for biogeographic reconstruction. An interactive map with all referenced localities is available online (Schönhofer 2013b).

Applying the extant distribution of species and the species tree derived from *BEAST analyses, the Vicariance Inference Program (VIP, Arias 2010; Arias et al. 2011) was used to infer the distribution of clades and distributional barriers. This program reconstructs the probability of distributional barriers using parsimony. For area reconstruction in VIP the grid was set to 0.3 without fill. To account for misidentifications, a maximum area overlap of $5 \%$ was allowed between species. A heuristic search was conducted using 1000 iterations, setting full sector search and otherwise using default settings.

\section{RESULTS}

Molecular phylogeny.-A total of 39 Ischyropsalis specimens were included in molecular phylogenetic analyses, with samples available for all described subspecies, and 18 of 22 currently described species. Previously published sequences were included from GenBank for I. luteipes, I. robusta Simon 1872 and I. nodifera Simon 1879 (Shultz \& Regier 2001; Schönhofer \& Martens 2010b; Giribet et al. 2010; see Appendix). Outgroup sequences for the sister genera Ceratolasma Goodnight \& Goodnight 1942 and Acuclavella Shear 1986 were obtained from Richart \& Hedin (2013).

The protein alignments of $\mathrm{CO} 1$ and the EFI $\alpha$ exon contained no gaps and no stop codons. We note that although EFI $\alpha$ paralogs have been detected in mite harvestmen (Clouse et al. 2013), we found no evidence for paralogs in Ischyropsalis. PCR products directly Sanger sequenced showed no evidence for multiple overlapping sequences as would be expected if paralogs were common. However, this result is potentially biased by the PCR primers that we utilized. As such, we also searched for EFI $\alpha$ paralogs in the transcriptomes of other ischryopsalidoid taxa (Hedin et al. 2012; unpublished data). Specifically, we blasted Ischyropsalis EFI $\alpha$ sequences (I. carli Lessert 1905, AXLS 145) against the transcriptomes of Sabacon Simon 1879, Acuclavella and Hesperonemastoma Gruber 1970 (tblastx, e value 1e-20) in Geneious 7.0.6. These searches recovered a single EFI $\alpha$ sequence for each of these ischryopsalidoid taxa, suggesting a lack of paralogy in this clade. We emphasize that Opiliones is an old taxon with ancient internal divergences (Hedin et al. 2012), and that gene duplication in one lineage does not imply similar molecular evolutionary patterns in other, distantly related taxa. Similar dynamics of EFI $\alpha$ paralogy gain and loss are observed, for example, in insects (Djernaes \& Damgaard 2006).

Models of sequence evolution were selected as follows: 28SrRNA: GTR+I+G; COI codon position 1: SYM+G; COI codon position 2: $\mathrm{F} 81+\mathrm{G}$; $\mathrm{COI}$ codon position 3: $\mathrm{GTR}+\mathrm{G}$; EFI $\alpha$ codon position 1: $\mathrm{HKY}+\mathrm{I}+\mathrm{G}$; $\mathrm{EFI} \alpha$ codon position 2: HKY; EFI $\alpha$ codon position 3: GTR+G; $\mathrm{EFI} \alpha$ intron: $\mathrm{HKY}+\mathrm{G}$. In *BEAST settings were modified for EFI $\alpha$-exon to $\mathrm{SYM}+\mathrm{I}+\mathrm{G}$ (in *BEAST set to $\mathrm{TN} 93+\mathrm{I}+\mathrm{G}$, base frequencies 
equal) and for COI to GTR $+\mathrm{I}+\mathrm{G}$ (same in *BEAST), both separated by codon.

Ischyropsalis is clearly monophyletic with respect to the North American Acuclavella and Ceratolasma, allowing removal of these outgroups for further reconstructions. Results of the concatenated Bayesian and ML analyses, both with and without outgroups, were generally congruent (Fig. 3), with the few topological differences observed at weakly supported nodes. Alternative topologies recovered different arrangements between Ischyropsalis lithoclasica Schönhofer \& Martens 2010a, I. kollari and I. ravasinii, and grouped I. navarrensis Roewer 1950 with I. robusta in the ML analyses. The same was true for the position of terminals within the two I. luteipes clades and I. carli (Fig. 3).

Bayesian and ML molecular phylogenetic results indicate that Ischyropsalis is divided into three primary clades, defined here for further discussion: 1) the I. hellwigii group (comprising the two described subspecies), 2) the I. manicata group (including I. adamii, I. carli, I. manicata L. Koch 1869 and I. pyrenaea alpinula Martens 1978), and 3) the Iberian-Alpine group, including all remaining sampled species. The composition of these groups is further detailed in the Discussion. Molecular phylogenetic support for these clades is strong (e.g., Bayesian posterior probability values $>0.95$ ), although slightly lower for the I. manicata group, which includes several species that are missing data for multiple genes (Appendix).

Bayesian and ML concatenated analyses (Fig. 3) recovered most of the currently accepted species as well-supported lineages, with some exceptions. The subspecies of Ischyropsalis pyrenaea are not recovered as monophyletic, but instead fall into different higher-level groups. Ischyropsalis luteipes was found to be paraphyletic with I. pyrenaea pyrenaea nested within I. luteipes. Intraspecific genetic divergences were generally low, except for I. adamii and I. dentipalpis, both including divergent genetic lineages. Distinct lineages within the non-monophyletic or internally-divergent taxa were defined as distinct OTUs in the *BEAST analysis of the diversified species set, and all received strong support (Fig. 4). However, the Bayes Factor analysis did not conclusively favor one species-set hypothesis over the alternative hypothesis (ln Bayes Factor: 3.635).

Biogeographic analysis.-VIP results are visualised in Figs. 5 and 6, including distributions of all higher clades (Fig. 5) and the Iberian-Alpine-group (Fig. 6). VIP recognized thirteen barriers corresponding to phylogenetic splits. Barriers were found in consistent positions over all reconstructions, except for a few cases when individual VIP reconstructions dropped taxa, which shifted the barrier in one direction towards one of the included groups but never changed its directionality or general position.

Biogeographic analyses indicate few putative long-range dispersal events between different European mountain systems within the I. hellwigii and I. manicata groups. The IberianAlpine group showed a general pattern of diversification coinciding with the division of ancestral areas, resulting in decreasing distribution areas with decreasing phylogenetic age of the clades, with evidence for long-range dispersal generally absent. Thus geographic proximity generally coincides with closer phylogenetic relationship within the Iberian-Alpine group (Fig. 6). Species distributions within the Iberian-Alpine group are not strictly allopatric but overlap in many cases, and in the Iberian distributional area at least two sympatric sister species are present.

\section{DISCUSSION}

Higher-level phylogeny. - Comparing phylogenetic hypotheses (Fig. 2) of Martens (1969b) and Dresco (1970) with molecular-based concatenated (Fig. 3) or *BEAST trees (Fig. 4) reveals little congruence. These alternative hypotheses agree only in the separate (Martens 1969b; Dresco 1970) and basal placement (Martens 1969b) of the I. hellwigii group. Further similarities only partly coincide with para- and polyphyletic groups, except for Hadži's Odontopalpa, that receives weak support in our analyses. Hadži (1931) included the species I. kollari (sub I. triglavensis) and I. dentipalpis (presently split into I. lithoclasica and I. dentipalpis sensu stricto; Schönhofer \& Martens 2010a) in this subgenus and assumed I. ravasinii to be included, although he had no males to confirm this placement. Martens (1969b) considered the genus Ischyropsalis too small to justify establishment of subgenera. Accepting Odontopalpa would require the definition of several additional subgenera, which he felt carried little taxonomic value - we generally agree with this recommendation.

While the detailed studies of previous authors refined the characters for species-level taxonomy, higher-level groups (as implied by molecular phylogeny) now lack morphologically diagnostic characters. This situation is similar to other harvestmen groups, for example Trogulus Latreille 1802, where characters delineating species appear clearer than those characterizing higher-level groups (Schönhofer \& Martens 2010b). The molecular results for Ischyropsalis suggest that most of the presently used morphological systematic characters are randomly distributed throughout the phylogeny. Even male-specific characters of the genitalia and cheliceral apophyses seem problematic for use as phylogenetic characters. This is not completely unexpected as Martens (1969b) mentioned the relatively simple organization of the penis in Ischyropsalis, perhaps prone to homoplasy or morphological stasis.

Character conflict is well-illustrated by the "subspecies" of I. pyrenaea, previously believed to be closely related based on morphology, but apparently belonging to different groups within Ischyropsalis (Fig. 3). The proposed shared characters of the I. pyrenaea subspecies (Martens 1969b, 1978), including cheliceral spination and apophyses, the male cheliceral bristle field and the overall similarity of the male genital morphology (e.g., un-lobed and keeled sclerites of the penial glans), are likely convergent. Indeed, the structure of these sclerites differs considerably in detail, particularly in the connection towards the sclerotised part of the penial stylus (Fig. 1). In I. pyrenaea pyrenaea this sclerite is rather broad, while in I. pyrenaea alpinula it is extremely narrow, appearing almost petiolate.

An overview of characters for diagnosing recovered molecular clades is given below. Ischyropsalis hadzii Roewer 1950, I. gigantea Dresco 1968, I. petiginosa Simon 1913 and I. cantabrica Luque \& Labrada 2012, not included in our data set, are also assigned tentatively to these larger groups.

I) I. hellwigii group (two described subspecies I. h. hellwigii, I. h. lucantei Simon 1879): Chelicerae massive, males with 


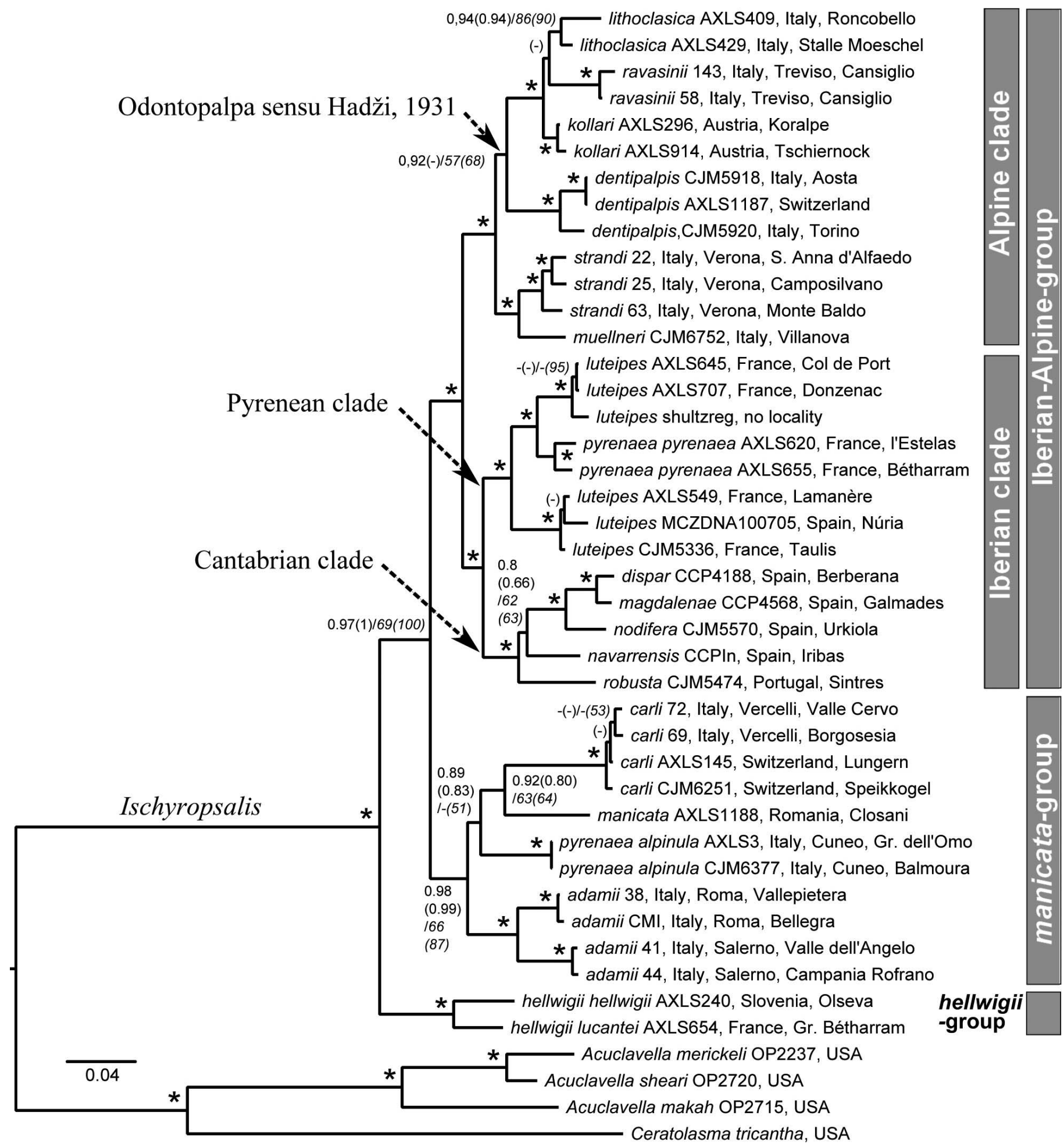

Figure 3.-Bayesian consensus phylogram based on concatenated molecular data. Numbers at nodes show support values resulting from different analyses: 1) Bayesian posterior probabilities; numbers in parentheses are Bayesian posterior probabilities after removal of outgroup genera and realignment of $28 \mathrm{~S}$ and $\mathrm{EF} 1 \alpha$ intron, 2) maximum likelihood bootstrap values (in italics); in parentheses after removal of outgroup and realignment of $28 \mathrm{~S}$ and EF1 $\alpha$ intron. Nodes with $\mathrm{pp}=1.00$ and bootstrap values $>94$ indicated by asterisks. Support values below 0.94 pp and 70 bootstrap not shown. 


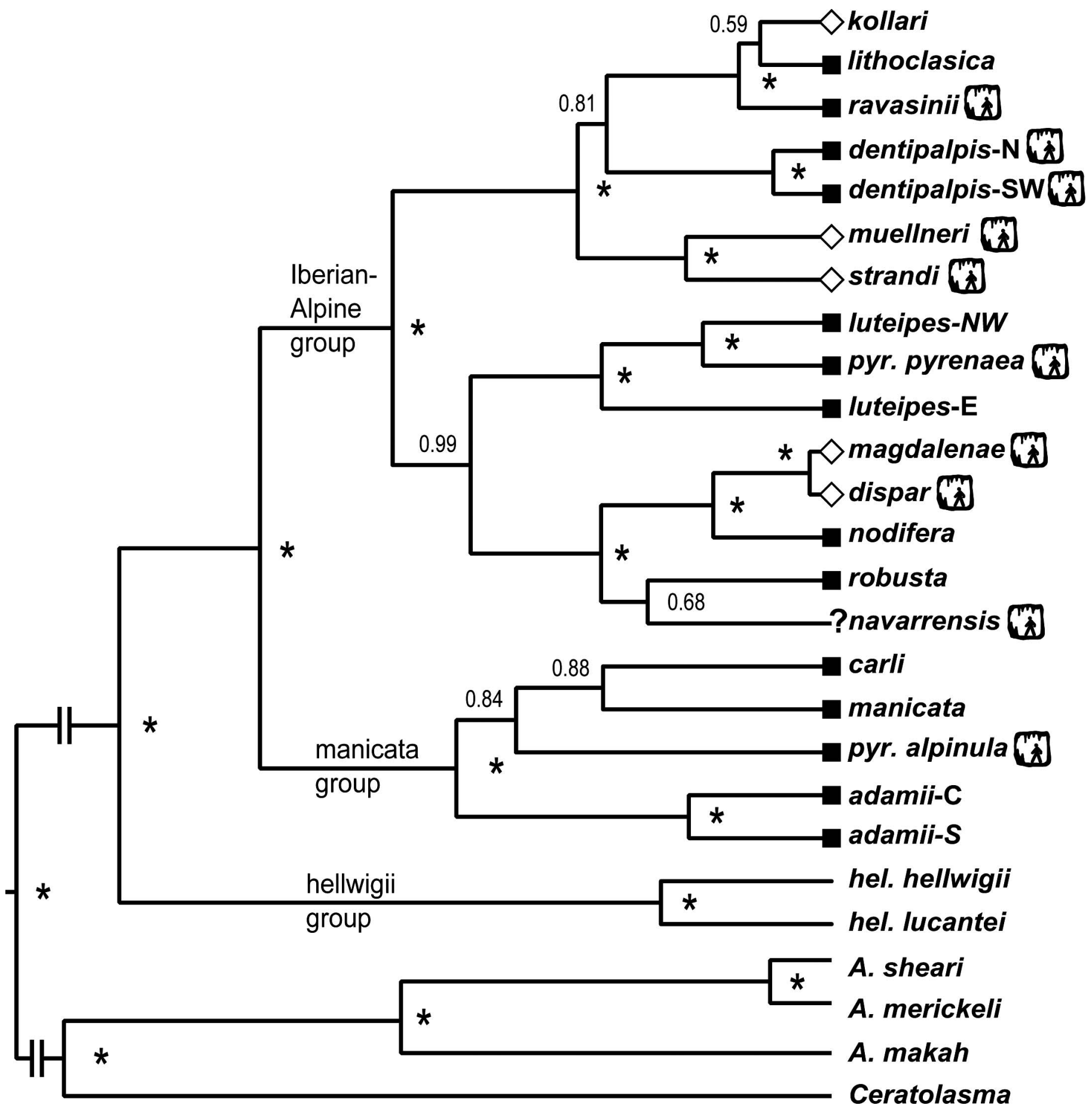

Figure 4.- *BEAST species tree. Basal nodes graphically shortened to allow expanded view of ingroup. Posterior probabilities given at nodes; pp of 1.00 indicated by an asterisk. Distribution of species group taxa according to Martens (1969b) is symbolized at tips: dentipalpis group indicated by black squares, kollari group indicated by open diamonds. I. lithoclasica was formerly considered I. dentipalpis, I. dentipalpis was considered I. helvetica (Martens 1969b; 1978), and I. pyrenaea alpinula was described as a subspecies by Martens (1978). Predominantly cavedwelling species indicated by a cave icon.

a large dorsal cheliceral glandular bristle field. Sclerite of penial gland small, with irregularly spaced small spines. Ischyropsalis $h$. lucantei is restricted to the central Pyrenees, while I. h. hellwigii occupies a large area from Germany to Southern Poland.
The following groups share long bristles on the penial glandes:

II) I. manicata group (I. adamii, I. carli, I. manicata, I. pyrenaea alpinula): Penial sclerites narrow-stalked, appearing almost narrow leaf- to broad heart-shaped. 


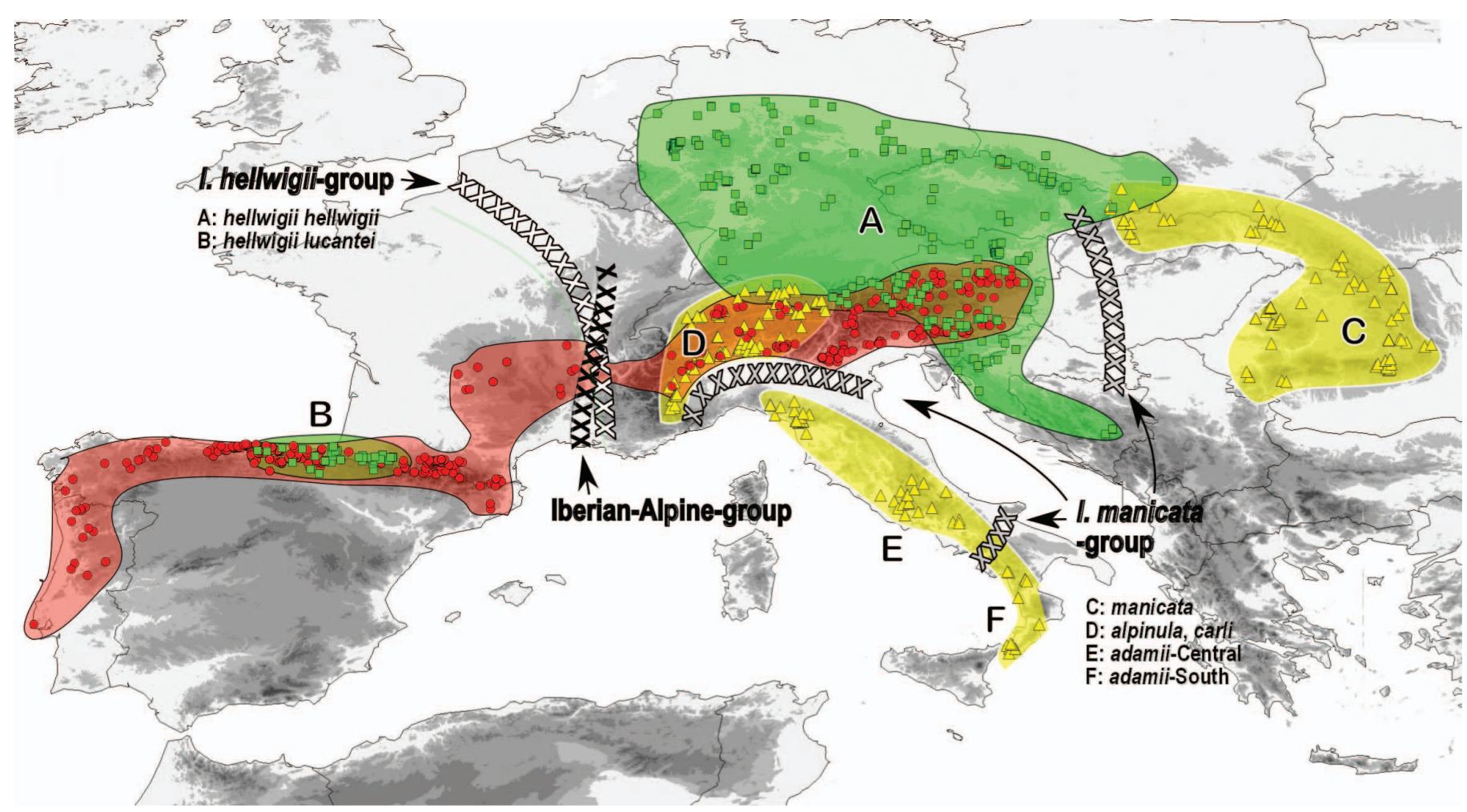

Figure 5.-Distribution of primary Ischyropsalis groups in Europe, with VIP barriers shown as lines of crosses. Major groups include the $I$. hellwigii (green squares), I. manicata (yellow triangles), and Iberian-Alpine (red circles) groups.

Sclerites always keeled and never bilobed, males with small distinctly circumscribed distolateral cheliceral glandular bristle field. Individual species have a rather widespread distribution. Two species in the western Alps (I. carli, I. pyrenaea alpinula), and one species in the Carpathians (I. manicata) and Apennines ( $I$. adamii), respectively; in the latter two areas these represent the only Ischyropsalis taxa known.

III) Iberian-Alpine group: Including the remaining species. Penial sclerites broad-stalked or not narrowed towards intersection with stylus. Otherwise morphologically heterogeneous and best separated by geographic distribution.

IIIa) Alpine-clade: the group is separated into two morphologically separable clades that share few characteristics, maybe the bilobed penial sclerite, which is not split in I. dentipalpis. Most species of the first group (I. dentipalpis, I. kollari, I. lithoclasica, I. muellneri Hamann 1898) exhibit a unique patellar apophysis on the male palp (missing in I. hadzii that likely belongs to this group as well) and have a large plateau-like bristle-field with sparse and scattered setation on the basal cheliceral segment (also in I. hadzii). Members of the second group within this clade ( $I$. ravasinii and I. strandi Kratochvíl 1936) lack the palpal apophyses and bristle field and apophyses are reduced or missing. Both groups are distributed mainly in the Central to Eastern Alps, with only I. dentipalpis in the Western Alps.
IIIb) Iberian-clade (including two subclades): many species have keeled penial sclerites, otherwise sclerites are always deeply bi-lobed. Males have large triangular dorsodistal cheliceral apophyses. Members of the Pyrenean clade (I. luteipes [two lineages], I. pyrenaea pyrenaea) share no perceivable characters, which highlights that most observed character states can evolve rapidly. Uniting features include distribution in the Pyrenees and molecular synapomorphies. The Cantabrian clade (I. dispar Simon 1872, I. magdalenae Simon 1881, I. navarrensis, I. nodifera, I. robusta, I. petiginosa, likely $I$. cantabrica and I. gigantea) has a primary uniting character, the bristle field on the basal cheliceral segment, situated on the distomedial side. The cheliceral apophysis is generally very large, often triangular and pointed, viewed laterally. This group is in need of further evaluation, including study of more populations, missing species and more refined geographic analyses.

Species-level systematics.-Our molecular phylogenetic results fail to recover two described species as monophyletic, or recover species as monophyletic but with high internal genetic divergence, perhaps consistent with cryptic or incipient speciation. As previously discussed the two subspecies of Ischyropsalis pyrenaea are distantly related (Figs. 3 \& 4). Considering their phylogenetic distance, they are hereby formally elevated to species (I. pyrenaea pyrenaea to I. pyrenaea and I. pyrenaea alpinula to I. alpinula). A careful redescription is warranted and may reveal further phyloge- 

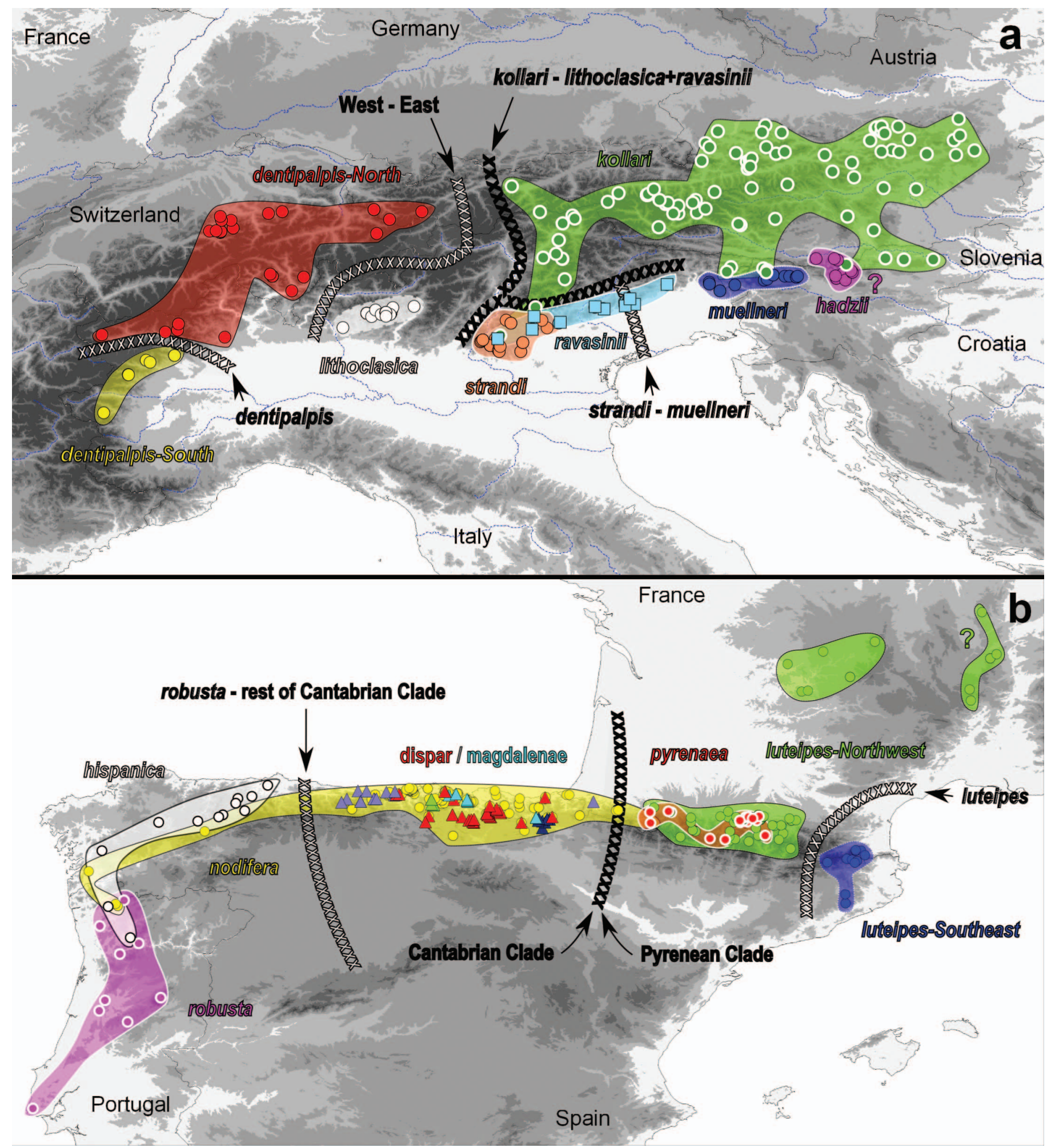

Figure 6.- a. Distribution of the Ischyropsalis Alpine clade in the Alps showing biogeographic reconstructions within major clades; b. Distribution map of the Ischyropsalis Iberian clade on the northern Iberian Peninsula and southern France. The distribution of microendemic species in the Cantabrian Mountains of northern Spain was not further resolved. For both a \& b, colors correspond to individual species and lineages as supported by *BEAST and other analyses, and VIP barriers are shown as lines of crosses. Question marks indicate populations or species where phylogenetic information is lacking. 
netically useful characters. Also in need of further study is the paraphyletic I. luteipes, with the morphologically different $I$. pyrenaea nested within this lineage (Fig. 3). Dresco (1970) discussed three "ecoforms" of I. luteipes but did not formally describe these or provide characters for delineation. Character reinvestigation and additional comprehensive geographic sampling is required.

The central and southern lineages of the Italian Ischyropsalis adamii are genetically clearly separable (Fig. 3), while material from the isolated northern population was not available for this study. Individual names for all three populations have been proposed by earlier authors and require reassessment (southern Italy: I. adamii; northern Italy: I. apuanus Caporiacco 1930; central Italy: I. apuanus nanus Dresco 1968). Similarly, our data indicate that the southwestern-most population of $I$. dentipalpis is genetically distinct. This species has been recently re-evaluated based on morphology (Schönhofer \& Martens 2010a), and further studies of its fragmented distribution were suggested. Our results indicate differentiation within I. dentipalpis but refute a close relationship to I. lithoclasica as hypothesized by Schönhofer and Martens (2010a).

Historical biogeography.-While Martens (1969b) assumed widespread species groups with strictly allopatric species, our phylogenetic results indicate a clearly different pattern. Herein, diversification and speciation is generally restricted to the same mountain range, specifically the Alps, the Cantabrian Mountains, the Pyrenees, and, to a lesser extent, the Apennines. Only in the monotypic Ischyropsalis hellwigii and I. manicata groups are species and lineages distantly allopatric, consistent with old vicariance or long-range dispersal (Fig. 5). The remaining Ischyropsalis species belonging to the Iberian-Alpine group are frequently sympatric, sometimes with completely overlapping distributions of closely related taxa, e.g., I. luteipes and I. pyrenaea. The biogeographic pattern within the Iberian-Alpine group shows a general trend of decreasing distribution area with decreasing taxonomic level, with the distribution of descendants contained within the distributional area of reconstructed ancestors. Therefore, dispersal seems not to be the main cause of diversification within Ischyropsalis, which remains difficult to test without reliably assignable fossils. The lack of Ischyropsalis fossils also means that we cannot rule out the presence of now extinct representatives in areas outside the known distribution of the genus.

Evolution of habitat preferences.-Most extant Ischyropsalis species are confined to montane habitats, preferring microhabitats with moderate to cold temperatures and high and constant humidity. On the Iberian Peninsula Ischyropsalis species are confined to mountain regions situated along the northern and western coast, characterised by high and consistent rainfall and moderate variation in annual temperatures (Immerzeel et al. 2009). Precipitation in the Alps is generally high, and low temperature microhabitats are abundant at high altitudes in this mountain chain.

It is noticeable that Ischyropsalis shows a general trend from more generalist and widespread species (I. hellwigii and I. manicata groups) towards specialized short-range endemics (most members of the Iberian-Alpine group). This trend manifests in the high number of cave endemics in Ischyr- opsalis, which may be considered extreme ecological specialists. Molecular phylogenetic trees include no large clades of exclusively cave-dwelling taxa (Fig. 4). Rather, it appears that cave species have evolved multiple times in clades of mainly epigean species, a pattern reported from many other cave radiations (Porter 2007), including harvestmen (Derkarabetian et al. 2010; Hedin \& Thomas 2010; Derkarabetian \& Hedin 2014). However, the degree of cave specialization in Ischyropsalis is variable, with many surface-dwelling species regularly entering caves. Caves are the only available habitats at low altitudes, with surface habitats such as alpine gravel or forests increasing towards high altitudes. Fully troglomorphic taxa are rare, as most species retain eyes. The tendency towards troglomorphism correlates with a preference for cooler and more stable temperatures. For example, temperature preferences for the surface-dwelling I. luteipes were recorded at $11.5{ }^{\circ} \mathrm{C}$ with a range of $10{ }^{\circ} \mathrm{C}$ (Juberthie 1964). In the troglomorphic I. pyrenaea pyrenaea the mean temperature is comparable, but the range does not exceed $5^{\circ} \mathrm{C}$. A similar preference range is exhibited by eastern Alpine cave endemics but with mean preferences shifted to even lower temperatures (I. strandi: $3.5-7.2{ }^{\circ} \mathrm{C}$; I. muellneri: $5.2-7.8{ }^{\circ} \mathrm{C}$; Juberthie 1964). The frequent evolution of cave specialization enables the regional co-occurrence of epigean and predominantly troglomorphic species. The preference for low temperatures might also explain the general absence of Ischyropsalis in more southern cave systems as on the Balkan, as these caves generally show higher mean temperatures.

Members of the Iberian-Alpine group and all cave endemic species may be vulnerable to climate change given their geographically restricted ranges and narrow ecological preferences. Considering these factors, it will be important to protect relevant cave habitats as comprehensively as possible.

\section{ACKNOWLEDGMENTS}

We are grateful to all colleagues who provided material for molecular studies, including Siegfried Huber (Unteruhldingen, Germany), Marco Isaia (Turin, Italy), Enrico Lana (Verolengo, Italy), Tone Novak (Slovenj Gradec, Slovenia), Rodica Plaiasu (Bucharest, Romania), Matteo Girardi (FEM, Trento, Italy) Claudio Chemini (Trento, Italy), Leonardo Latella (Verona, Italy), Valeria Lencioni (Trento, Italy) and Carlos Prieto (Bilbao, Spain). Clemens Brandstaedter (Buers, Austria) and the German Spider-Forum (http://spinnen-forum.de/smf/index.php?board= 3.0) are thanked for their help with reconstructing problematic localities. Casey Richart (San Diego, USA) provided outgroup sequences. Salvador Arias (Tucumán, Argentina) provided help with running the VIP analysis. A.L.S. was funded by a postdoctoral grant of the Deutsche Forschungsgemeinschaft (DFG) to work on systematics and biogeography of Holarctic Opiliones. J.M. was regularly sponsored by Feldbausch Foundation and Wagner Foundation, both at Fachbereich Biologie, Mainz University to carry out field work. Molecular research in the Hedin lab was supported by a California State University Program for Education and Research in Biotechnology (CSUPERB) grant to M. Hedin. Comments of two anonymous reviewers helped to improve the manuscript.

\section{LITERATURE CITED}

Arias, J.S. 2010. VIP. Online at http://www.zmuc.dk/public/phylogeny/ $\mathrm{vip} /$ (last accessed 03.12.2014). 
Arias, J.S., C.A. Szumik \& P.A. Goloboff. 2011. Spatial analysis of vicariance: a method for using direct geographical information in historical biogeography. Cladistics 27:1-12.

Boyer, S.L. \& C.N. Reuter. 2012. New species of mite harvestmen from the Wet Tropics of Queensland, Australia, with commentary on the biogeography of the genus Austropurcellia (Opiliones, Cyphophthalmi, Pettalidae). Journal of Arachnology 40:96-112.

Clouse, R.M., P.P. Sharma, G. Giribet \& W.C. Wheeler. 2013. Elongation factor- $1 \alpha$, a putative single-copy nuclear gene, has divergent sets of paralogs in an arachnid. Molecular Phylogenetics \& Evolution 68:471-481.

Derkarabetian, S. \& M. Hedin. 2014. Integrative taxonomy and species delimitation in harvestmen: A revision of the western North American genus Sclerobunus (Opiliones: Laniatores: Travunioidea). Public Library of Science ONE 9:e104982.

Derkarabetian, S., J. Ledford \& M. Hedin. 2011. Genetic diversification without obvious genitalic morphological divergence in harvestmen (Opiliones, Laniatores, Sclerobunus robustus) from montane sky islands of western North America. Molecular Phylogenetics and Evolution 61:844-853.

Derkarabetian, S., D.B. Steinmann \& M. Hedin. 2010. Repeated and time-correlated morphological convergence in cave-dwelling harvestmen (Opiliones, Laniatores) from montane western North America. Public Library of Science ONE 5:1-13.

Djernaes, M. \& J. Damgaard. 2006. Exon-intron structure, paralogy and sequenced regions of Elongation Factor-1 alpha in Hexapoda. Arthropod Systematics \& Phylogeny 64:45-52.

Dresco, E. 1970. Recherches sur la variabilité et la phylogénie chez les opilions du genre Ischyropsalis C.L. Koch (Fam. Ischyropsalidae), avec création de la famille nouvelle des Sabaconidae. Bulletin du Museum National d'Histoire Naturelle 2e Serie 41:1200-1213.

Drummond, A.J., M.A. Suchard, D. Xie \& A. Rambaut. 2012. Bayesian phylogenetics with BEAUti and the BEAST 1.7. Molecular Biology \& Evolution 29:1969-1973.

Geneious 7.0.6 created by Biomatters. Online at http://www.geneious. com/

Giribet, G., L. Vogt, A. Perez Gonzalez, P. Sharma \& A.B. Kury. 2010. A multilocus approach to harvestman (Arachnida: Opiliones) phylogeny with emphasis on biogeography and the systematics of Laniatores. Cladistics 26:408-437.

Gruber, J. 1998. Beiträge zur Systematik der Gattung Dicranolasma (Arachnida: Opiliones, Dicranolasmatidae). I. Dicranolasma thracium STAREGA und verwandte Formen aus Südosteuropa und Südwestasien. Annalen des Naturhistorischen Museums in Wien, B, 100:489-537.

Grummer, J.A., R.W. Bryson Jr. \& T.W. Reeder. 2014. Species delimitation using Bayes factors: Simulations and application to the Sceloporus scalaris species group (Squamata: Phrynosomatidae). Systematic Biology 63:119-133.

Hadži, J. 1931. Opilioni Triglavskoga masiva [German title from abstract: Die Opilionen des Triglavmassivs]. Prirodoslovne Razprave (Muzejsko Drustvo za Slovenijo). Ljubljana 1:107-154.

Hedin, M. \& S.M. Thomas. 2010. Molecular systematics of eastern North American Phalangodidae (Arachnida: Opiliones: Laniatores), demonstrating convergent morphological evolution in caves. Molecular Phylogenetics \& Evolution 54:107-121.

Hedin, M., S. Derkarabetian, M. McCormack, C. Richart \& J.W. Shultz. 2010. The phylogenetic utility of the nuclear protein-coding gene EF-1a for resolving recent divergences in Opiliones, emphasizing intron evolution. Journal of Arachnology 38:9-20.

Hedin, M., J. Starrett, S. Akhter, A.L. Schönhofer \& J.W. Shultz. 2012. Phylogenomic resolution of Paleozoic divergences in harvestmen (Arachnida, Opiliones) via analysis of next-generation transcriptome data. Public Library of Science ONE 7:e42888.

Heled, J. \& A.J. Drummond. 2010. Bayesian inference of species trees from multilocus data. Molecular Biology \& Evolution 27:570-580.
Helversen, O. von. \& J. Martens. 1972. Unrichtige Fundort-Angaben in der Arachniden-Sammlung Roewer. Senckenbergiana Biologica 53:109-123.

Huelsenbeck, J.P. \& F. Ronquist. 2001. MRBAYES: Bayesian inference of phylogeny. Bioinformatics 17:754-755.

Immerzeel, W.W., M.M. Rutten \& P. Droogers. 2009. Spatial downscaling of TRMM precipitation using vegetative response on the Iberian Peninsula. Remote Sensing of Environment 113:362-370.

Juberthie, C. 1964. Morphologie et biologie comparée de quelques opilions du genre Ischyropsalis C. L. K. Spelunca Mémoires 4:181-184.

Katoh, K. \& H. Toh. 2008. Improved accuracy of multiple ncRNA alignment by incorporating structural information into a MAFFTbased framework. BMC Bioinformatics 9:1-13.

Luque, C.G. 1991. Los Ischyropsalidoidea de la Cornisa Cantábrica: Cantabria. Arquenas, Fauna Iberica Subterránea: Cantabria $1: 1-96$.

Luque, C.G. 1992. Contribución al conocimiento de los Opiliones epigeos e hipogeos de Cantabria (España). Primeras aportaciones al catálogo (Arachnida, Opiliones, Palpatores). Proceedings 5th Spanish Meeting of Speleology 1:143-157.

Luque, C.G. \& L. Labrada. 2012. A new cave-dwelling endemic Ischyropsalis C.L. Koch, 1839 (Opiliones: Dyspnoi: Ischyropsalididae) from the karstic region of Cantabria (Spain). Zootaxa 3506:26-42.

Mallatt, J. \& J. Sullivan. 1998. 28S and 18S rDNA sequences support the monophyly of lampreys and hagfishes. Molecular Biology \& Evolution 15:1706-1718.

Martens, J. 1969a. Die Sekretdarbietung während des Paarungsverhaltens von Ischyropsalis C.L. Koch (Opiliones). Zeitschrift fur Tierpsycholologie 26:513-523.

Martens, J. 1969b. Die Abgrenzung von Biospezies auf biologischethologischer und morphologischer Grundlage am Beispiel der Gattung Ischyropsalis C.L. Koch 1839 (Opiliones, Ischyropsalididae). Zoologische Jahrbücher, Jena, Abteilung für Systematik, Ökologie und Geographie der Tiere 96:133-264.

Martens, J. 1972. Opiliones aus dem Nepal-Himalaya. I. Das Genus Sabacon Simon (Arachnida: Ischyropsalididae). Senckenbergiana Biologica 53:307-323.

Martens, J. 1978. Spinnentiere, Arachnida-Weberknechte, Opiliones. Tierwelt Deutschlands 64:1-464.

Martens, J. \& W. Schawaller. 1977. Die Cheliceren-Drüsen der Weberknechte nach rasteroptischen und lichtoptischen Befunden (Arachnida: Opiliones). Zoomorphologie 86:223-250.

Mayr, E. 1942. Systematics and the Origin of Species from the Viewpoint of a Zoologist. Columbia University Press, New York.

Porter, M. 2007. Subterranean biogeography: what have we learned from molecular techniques? Journal of Cave \& Karst Studies 69:179-186.

Posada, D. 2008. JModelTest: phylogenetic model averaging. Molecular Biology \& Evolution 25:1253-1256.

Prieto, C.E. 1990a. The genus Ischyropsalis C.L. Koch (Opiliones, Ischyropsalididae) on the Iberian Peninsula. I. Non-troglobitic species. Acta Zoologica Fennica 190:315-320.

Prieto, C.E. 1990b. The genus Ischyropsalis C.L. Koch (Opiliones, Ischyropsalididae) on the Iberian Peninsula. II. Troglobitic species. Bulletin de la Société Européenne d'Arachnologie, $\mathrm{N}^{\circ}$ hors série 1:286-292.

Rambaut, A. \& A.J. Drummond. 2007. Tracer v1.4. Online at http:// beast.bio.ed.ac.uk/Tracer (last accessed 03.12.2014).

Richart, C.H. \& M. Hedin. 2013. Three new species in the harvestmen genus Acuclavella (Opiliones, Dyspnoi, Ischyropsalidoidea), including description of male Acuclavella quattuor (Shear 1986). Zookeys 311:19-68.

Roewer, C.F. 1950. Über Ischyropsalididae und Trogulidae. Weitere Weberknechte XV. Senckenbergiana 31:11-56. 
Ronquist, F., J.P. Huelsenbeck \& M. Teslenko. 2011. MrBayes 3.2 Draft MrBayes version 3.2 Manual: Tutorials and Model Summaries. Online at $\mathrm{http}: / / \mathrm{mrb}$ bes.sourceforge.net $/ \mathrm{mb3}$.2_manual.pdf (last accessed 03.12.2014).

Schönhofer, A.L. 2013a. A taxonomic catalogue of the Dyspnoi Hansen and Sørensen, 1904 (Arachnida: Opiliones). Zootaxa 3679:1-68.

Schönhofer, A.L. 2013b. Distribution of the genus Ischyropsalis. Online at http://axelschoenhofer.weebly.com/ischyropsalis-distribution.html (last accessed 03.12.2014).

Schönhofer, A.L. \& J. Martens. 2010a. On the identity of Ischyropsalis dentipalpis Canestrini, 1872 and description of Ischyropsalis lithoclasica sp. n. (Opiliones: Ischyropsalididae). Zootaxa 2613:1-14.

Schönhofer, A.L. \& J. Martens. 2010b. Hidden Mediterranean diversity: Assessing species taxa by molecular phylogeny within the opilionid family Trogulidae (Arachnida, Opiliones). Molecular Phylogenetics \& Evolution 54:59-75.

Schönhofer, A.L. \& J. Martens. 2012. The enigmatic Alpine opilionid Saccarella schilleri gen. n., sp. n. (Arachnida: Nemastomatidae)isolated systematic placement inferred from comparative genital morphology. Organisms Diversity \& Evolution 12:409-419.

Schönhofer, A.L., M. McCormack, N. Tsurusaki, J. Martens \& M. Hedin. 2013. Molecular phylogeny of the harvestmen genus Sabacon (Arachnida: Opiliones: Dyspnoi) reveals multiple Eocene-Oligocene intercontinental dispersal events in the Holarctic. Molecular Phylogenetics \& Evolution 66:303-315.

Sharma, P.P. \& G. Giribet. 2012. Out of the Neotropics: Late Cretaceous colonization of Australasia by American arthropods.
Proceedings of the Royal Society of London B: Biological Sciences 279:3501-3509.

Shultz, J.W. \& J.C. Regier. 2001. Phylogenetic analysis of Phalangida (Arachnida, Opiliones) using two nuclear protein-encoding genes supports monophyly of Palpatores. Journal of Arachnology 29: 189-200.

Shultz, J.W. \& J.C. Regier. 2009. Caddo agilis and C. pepperella (Opiliones, Caddidae) diverged phylogenetically before acquiring their disjunct, sympatric distributions in Japan and North America. Journal of Arachnology 37:238-240.

Silvestro, D. \& I. Michalak. 2012. raxmlGUI: a graphical front-end for RAxML. Organisms Diversity \& Evolution 12:335-337.

Stamatakis, A. 2006. RAxML-VI-HPC: maximum likelihood-based phylogenetic analyses with thousands of taxa and mixed models. Bioinformatics 22:2688-2690.

Suzuki, S. 1974. The Japanese species of the genus Sabacon (Arachnida, Opiliones, Ischyropsalididae). Journal of Science of the Hiroshima University, Series B, Division 1 (Zoology) 25:83-108.

Tamura, K., J. Dudley, M. Nei \& S. Kumar. 2007. MEGA4: Molecular Evolutionary Genetics Analysis (MEGA) software version 4.0. Molecular Biology \& Evolution 24:1596-1599.

Thomas, S.M. \& M. Hedin. 2008. Multigenic phylogeographic divergence in the paleoendemic southern Appalachian opilionid Fumontana deprehendor Shear (Opiliones, Laniatores, Triaenonychidae). Molecular Phylogenetics \& Evolution 46:645-658.

Vink, C.J., S.M. Thomas, P. Paquin, C.Y. Hayashi \& M. Hedin. 2005. The effects of preservatives and temperatures on arachnid DNA. Invertebrate Systematics 19:99-104.

Manuscript received 18 June 2014, revised December 62014. 


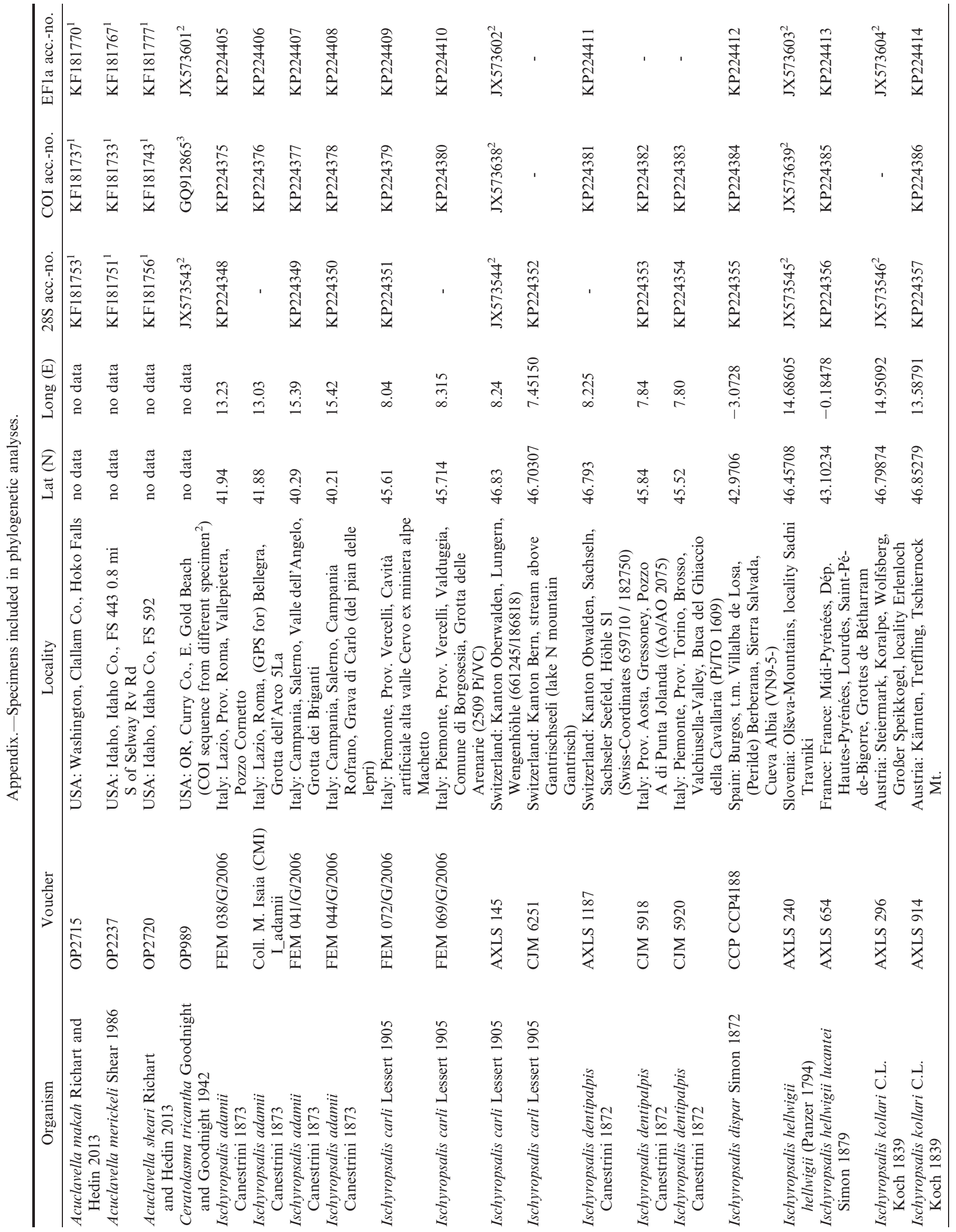




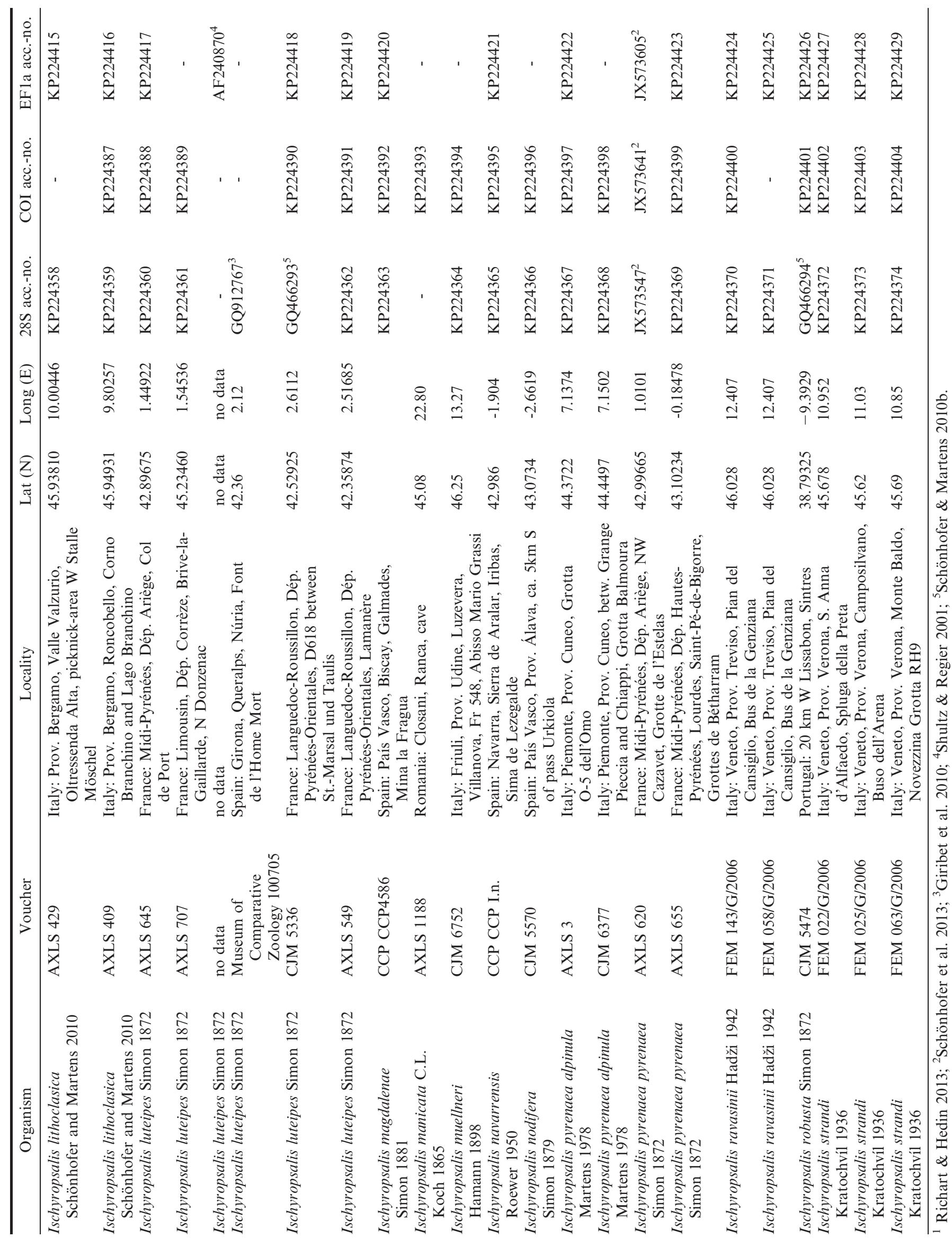

